1 Hacettepe Journal of Mathematics and Statistics

h Volume 47 (2) (2018), 219-235

\title{
Modelling the impact of climatic variables on malaria transmission
}

\author{
Gbenga J. Abiodun* ${ }^{* \dagger}$, P. Witbooi ${ }^{\ddagger}$ and Kazeem O. Okosun ${ }^{\S}$
}

\begin{abstract}
Malaria is one of the most severe disease in the world. The projected climate change will probably alter the region and transmission potential of malaria in Africa. In this study, a climate-based mathematical model to investigate the impact of temperature and rainfall on malaria transmission is developed and analysed. The basic reproduction number $\left(\mathcal{R}_{0}\right)$ is derived along with stability analysis. The effect of the larval death rate on the reproduction number is also investigated. The model is validated on observed malaria transmission in Limpopo Province, South Africa, giving a reasonable fit and in particular, detecting accurately all the spikes in malaria prevalence. The model provides a numerical basis for further refinement towards prediction of the impact of climate variability on malaria transmission.
\end{abstract}

Keywords: Malaria, climate, temperature, rainfall, basic reproduction number, disease-free equilibrium, endemic equilibrium

2000 AMS Classification: 49K15; 93D20

Received: 03.07.2016 Accepted: 13.02.2017 Doi : 10.15672/HJMS.2017.452

*Department of Mathematics and Applied Mathematics, University of the Western Cape, Private Bag X17, Bellville 7535, Republic of South Africa, Email: 3109433@myuwc.ac.za

${ }^{\dagger}$ Corresponding Author.

${ }^{\ddagger}$ Department of Mathematics and Applied Mathematics, University of the Western Cape, Private Bag X17, Bellville 7535, Republic of South Africa, Email: pwitbooi@uwc.ac.za

${ }^{\S}$ Department of Mathematics, Vaal University of Technology, Private Bag X021, Vanderbijlpark, Andries Potgieter Blvrd-1900, Republic of South Africa, Email: kazeemo@vut.ac.za 


\section{Introduction}

Malaria remains one of the largest killer diseases in Africa, and most of its victims are women and children [56]. Sub-Saharan Africa continues to carry an extremely high portion of the global malaria burden [57]. In South Africa, over $10 \%$ of the population are living in malaria-epidemic provinces and are posed to the danger of contacting the disease [36]. Malaria is caused by a protozoan from the genus Plasmodium, and it is spread through mosquitoes. A single bite by a malaria-carrying mosquito can lead to extreme sickness or death. Malaria starts with an extreme cold, followed by high fever and severe sweating. These can be accompanied by joint pain, abdominal pain, headaches, vomiting, and extreme fatigue.

Malaria is very sensitive to climatic conditions, which explains why it is most prevalent in tropical climates, where there is sufficient breeding sites and conducive temperatures for mosquitoes. The high sensitivity of malaria to climate cannot be over-emphasized. For instance, a slight change in temperature can drastically affect the lifespan and patterns of mosquitoes because they are cold-blooded, and moreover, the protozoan itself can only survive in certain temperatures. With higher temperatures, the mosquitoes can mature faster, and thus have more time to spread the disease. The malaria parasite also matures more quickly at warmer temperatures. However, if temperatures become too high, neither mosquitoes nor the malaria parasite can survive. In addition, water is also a major contributor to the spread of malaria, since the vector mosquitoes breed in small pools of water. More rainfall increases the possible breading grounds for mosquito larvae, which eventually results in more vectors to spread the disease. With little rainfall, there are few places for the mosquitoes to breed.

When attempting to control the mosquito population, it is important to examine the weather parameters such as temperature and rainfall which are imperative in determining the disease epidemics. Having accurate seasonal climate forecasts of these parameters, it is possible to utilize malaria models that account for early warning systems in endemic regions [52]. These models can also be used to evaluate the possible changes in malaria prevalence regions under climate change scenarios [27]. Malaria transmission models play a significant role in understanding the dynamics of the disease [34] and have long been applied to assess the possible means of intervention [26, 31]. The dynamics have been investigated through deterministic models in many studies [9, 49] and some through stochastic models $[37,51]$. Some of these studies either neglect the impact of climate or incorporate it through the force-of-infection. For instance, Okosun and Makinde [39] derived and analyzed a model for the transmission of malaria disease that includes the class of individuals with drug resistance and treatment measures. These parameters were used to formulate optimal strategies for disease control in the population. The local stability of the disease free equilibrium and the existence of an endemic equilibrium were also established in the study.

Many studies have considered climate as a major factor of the disease epidemic. Craig et al. [10], for instance examined both seasonal case totals and seasonal changes in cases aligned with a series of climatic indicators obtained from three weather stations in KwaZulu-Natal, South Africa. In the study, linear regression analysis with several climate variables is used to verify that seasonal changes are important. Eckhoff et al. [14] also offered a new model for mosquito population dynamics with the effect of weather and impacts of multiple concurrent interventions. The model is set in large-scale individualbased simulation and results for local elimination of malaria are discussed. More dynamic models for vector life-cycle has been set up and designed to run on a local scale with treatment of water bodies. The Liverpool Malaria Model (LMM) has also been useful 
in forecasting and climate projection analysis [24, 25]. It includes the effect of temperature on the sporogonic and gonotrophic cycles, and clearly explains the parasite and egg growth stages with consideration of temperature impact on vector death rates [19]. Although the model was made to run over a regional scale, it did not embrace a clear representation of the surface hydrology, and egg laying rates were proportional to the 10-day rain rate. The updated version of LMM (that is $\mathrm{LMM}_{2010}$ ) which works on daily mean temperature and rainfall was later introduced to fill the lapses above, [15, 16]. In the previous study of Okosun and Makinde [39], the impact of climate on malaria transmission is not put into consideration. Hence this study aims to extend the work of Makinde and Okosun [39] by examining the impact of climate variability on malaria epidemics over Limpopo province, South Africa.

We begin by developing the SEIR malaria model with recovery class. We incorporate climate variables (rainfall and temperature) into the model and illustrate some of its basic properties in Section 2. The equilibrium points are determined in Section 3. Estimation of both climate-dependent and climate-independent parameters follows in Section 4 . We validate our model against malaria transmission in Limpopo Province, South Africa in Section 5. Finally, concluding remarks appear in Section 6.

\section{Methodology}

2.1. Model formulation. Our model sub-divides the total human population, denoted by $N_{h}$ into sub-populations of susceptible individuals $\left(S_{h}\right)$, those exposed to malaria parasite $\left(E_{h}\right)$, individuals with malaria symptoms $\left(I_{h}\right)$ and recovered humans $\left(R_{h}\right)$ as illustrated in Fig. 1, such that $N_{h}=S_{h}+E_{h}+I_{h}+R_{h}$.

The total vector (mosquito) population is denoted by $N_{v}$ and is sud-divided into susceptible mosquitoes $\left(S_{v}\right)$, mosquitoes exposed to malaria parasite $\left(E_{v}\right)$ and infectious mosquitoes $\left(I_{v}\right)$. Hence, $N_{v}=S_{v}+E_{v}+I_{v}$.

Susceptible individuals are recruited (by birth or immigration) into the community at a rate $\Phi_{h}$ and acquire malaria through contact with infectious mosquitoes at a rate $\beta_{h}$. Exposed individuals move to infectious class at a rate $\eta_{h}$ while infected individuals recover spontaneously at a rate $\alpha$ to join the immune class as some recovered due to treatment at a rate $\varsigma$. Recovered individuals lose their immunity and return to susceptible class at a rate $q$ while the natural death and disease-induced death rate are denoted by $\mu_{h}$ and $\gamma$ respectively.

Susceptible mosquitoes $\left(S_{v}\right)$ are recruited at the rate $\vartheta_{v}(T, R) N_{v}\left(1-\frac{N_{v}}{P}\right)\left(1-\delta_{v}\right)$, where $P$ is the larval carrying capacity and $\delta_{v}$ represent the proportion of larvae that died in the process of becoming adult mosquito. We assume that larvae become adult at the rate $\vartheta_{v}(T, R)$ which is dependent on temperature $(T)$ and rainfall $(R)$. Mosquitoes are assumed to suffer death due to natural causes at a rate $\mu_{v}(T)$ and their numbers are further reduced due to the use of insecticide spray at a rate $\varrho$.

The susceptible mosquitoes acquire malaria through contact with infected humans at a rate $\xi_{v}$ and move to the exposed class $\left(E_{v}\right)$, later to progress towards the infected class $\left(I_{v}\right)$ at a rate $\eta_{v}(T)$. It is noted that $\beta_{h}=\frac{v \epsilon \kappa I_{v}}{N_{h}}$ and $\xi_{v}(T)=\frac{\xi \epsilon(T) \kappa I_{h}}{N_{h}}$, where $v$ and $\xi$ represent the transmission probability of human and mosquito respectively, with contact rate $\kappa$ of mosquito per human per unit time. We also assume that mosquito biting rate $\epsilon(T)$ and mortality rate $\mu_{v}(T)$ are temperature dependent $[4,38,53]$.

The dynamics of the disease are described by the following system of differential equations: 


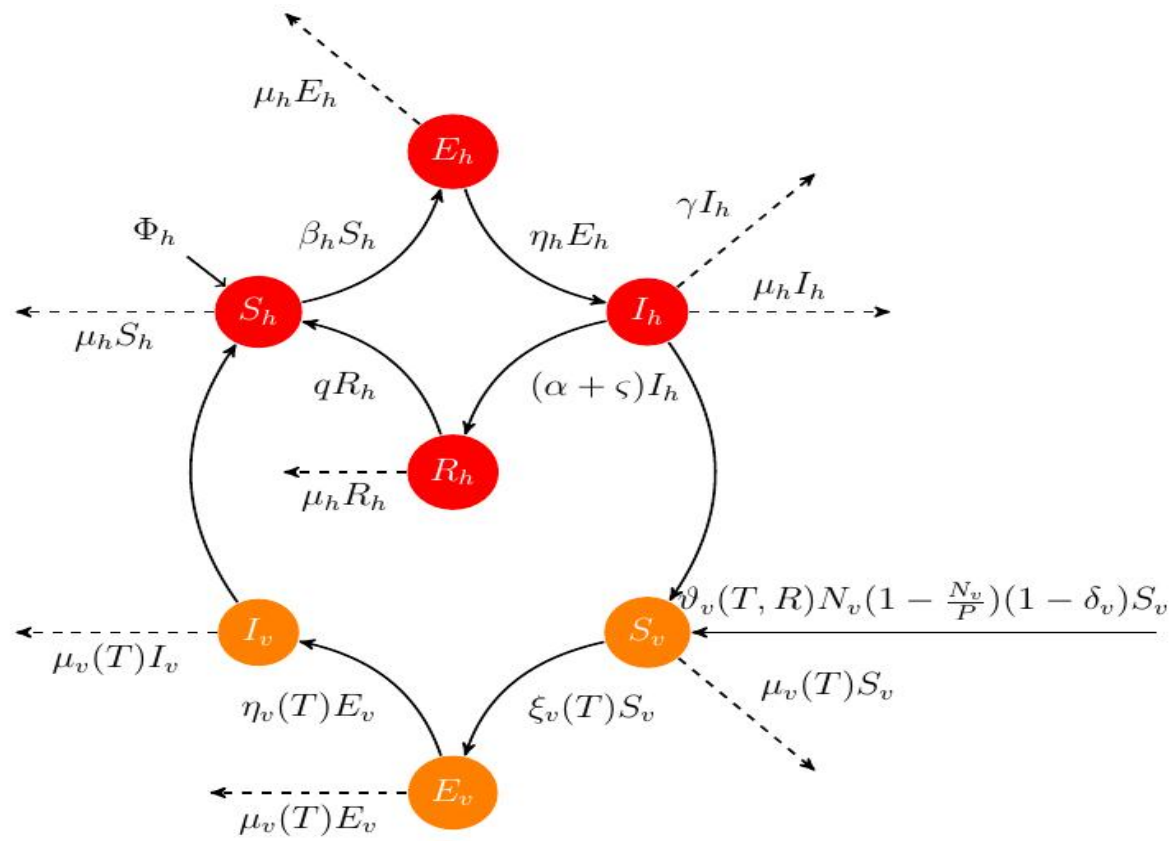

Figure 1. Flow diagram for malaria transmission model

$$
\begin{aligned}
& \frac{d S_{h}}{d t}=\Phi_{h}+q R_{h}-\beta_{h} S_{h}-\mu_{h} S_{h} \\
& \frac{d E_{h}}{d t}=\beta_{h} S_{h}-\left(\eta_{h}+\mu_{h}\right) E_{h} \\
& \frac{d I_{h}}{d t}=\eta_{h} E_{h}-\left(\alpha+\gamma+\varsigma+\mu_{h}\right) I_{h} \\
& \frac{d R_{h}}{d t}=(\alpha+\varsigma) I_{h}-\left(q+\mu_{h}\right) R_{h} \\
& \frac{d S_{v}}{d t}=\vartheta_{v}(T, R) N_{v}\left(1-\frac{N_{v}}{P}\right)\left(1-\delta_{v}\right)-\left(\xi_{v}+\varrho+\mu_{v}(T)\right) S_{v} \\
& \frac{d E_{v}}{d t}=\xi_{v} S_{v}-\left(\eta_{v}(T)+\varrho+\mu_{v}(T)\right) E_{v} \\
& \frac{d I_{v}}{d t}=\eta_{v}(T) E_{v}-\left(\varrho+\mu_{v}(T)\right) I_{v}
\end{aligned}
$$

Hence,

$$
\begin{aligned}
& \frac{d N_{h}}{d t}=\Phi_{h}-\gamma I_{h}-\mu_{h} N_{h} \\
& \frac{d N_{v}}{d t}=\vartheta_{v}(T, R) N_{v}\left(1-\frac{N_{v}}{P}\right)\left(1-\delta_{v}\right)-\left(\varrho+\mu_{v}(T)\right) N_{v} .
\end{aligned}
$$

2.2. Model analysis without climate parameters. In this section, we begin our mathematical analysis without climate-dependent parameters. We assume all parameters 
are constant. The SEIR malaria model (2.1) will be analyzed in a biologically feasible region as follows. This region should be feasible for both human and vector populations. More precisely, we have

2.1. Theorem. If $S_{h}(0), E_{h}(0), I_{h}(0), R_{h}(0), S_{v}(0), E_{v}(0)$ and $I_{v}(0)$ are non-negative, then so are $S_{h}(t), E_{h}(t), I_{h}(t), R_{h}(t), S_{v}(t), E_{v}(t)$ and $I_{v}(t)$ for all $t>0$. Moreover $\limsup _{t \rightarrow \infty} N_{h}(t) \leq \frac{\Phi_{h}}{\mu_{h}}$ and $\limsup _{t \rightarrow \infty} N_{v}(t) \leq P\left(1+\frac{\left(\varrho+\mu_{v}\right)}{\vartheta_{v}\left(1-\delta_{v}\right)}\right)$.

In addition, if $N_{h}(0) \leq \frac{\Phi_{h}}{\mu_{h}}$ and $N_{v}(0) \leq P\left(1+\frac{\left(\varrho+\mu_{v}\right)}{\vartheta_{v}\left(1-\delta_{v}\right)}\right)$ respectively, then $N_{h}(t) \leq \frac{\Phi_{h}}{\mu_{h}}$ and $N_{v}(t) \leq P\left(1+\frac{\left(\varrho+\mu_{v}\right)}{\vartheta_{v}\left(1-\delta_{v}\right)}\right)$ respectively. In particular, the region $D=D_{h} \times D_{v}$ with,

$$
D_{h}=\left\{\left(S_{h}, E_{h}, I_{h}, R_{h}\right) \in \mathbb{R}_{+}^{4}: S_{h}+E_{h}+I_{h}+R_{h} \leq \frac{\Phi_{h}}{\mu_{h}}\right\}
$$

and

$$
D_{v}=\left\{\left(S_{v}, E_{v}, I_{v}\right) \in \mathbb{R}_{+}^{3}: S_{v}+E_{v}+I_{v} \leq P\left(1+\frac{\left(\varrho+\mu_{v}\right)}{\vartheta_{v}\left(1-\delta_{v}\right)}\right)\right\}
$$

is positively invariant.

From this theorem we conclude that it is sufficient to consider the dynamics of the model in [35] on $D$. In this region, the model can be considered as being epidemiologically meaningful and mathematically well-posed $[1,18]$.

2.2.1. Existence and stability of equilibria.

Disease-free equilibrium (DFE). The basic model (2.1) has a DFE given by,

$$
\mathcal{E}_{0}=\left(S_{h}^{*}, E_{h}^{*}, I_{h}^{*}, R_{h}^{*}, S_{v}^{*}, E_{v}^{*}, I_{v}^{*}\right)=\left(\frac{\Phi_{h}}{\mu_{h}}, 0,0,0, P\left(1+\frac{\left(\varrho+\mu_{v}\right)}{\vartheta_{v}\left(1-\delta_{v}\right)}\right), 0,0\right)
$$

At this point we find it convenient to introduce the symbol $\bar{\mu}, \bar{\mu}=\alpha+\gamma+\varsigma+\mu_{h}$, in order to simplify a number of expressions. The linear stability of $\mathcal{E}_{0}$ is ascertained as in [54]. Using the next generation operator method on model (2.1) the basic reproduction number, denoted by $\mathcal{R}_{0}$, is found to be given by

$$
\mathcal{R}_{0}=r\left(F V^{-1}\right)=\sqrt{\mathcal{R}_{0 h} \times \mathcal{R}_{0 v}}
$$

where $r$ is the spectra radius (dominant eigenvalue in magnitude) of the next generation matrix $F V^{-1}$. The term $\mathcal{R}_{0 h}$ represents the number of humans that one mosquito infects through its infectious lifetime and it is defined as

$$
\mathcal{R}_{0 h}=\sqrt{\frac{v \epsilon \kappa \eta_{h} \mu_{h}}{\Phi_{h} \bar{\mu}\left(\eta_{h}+\mu_{h}\right)}}
$$

and $\mathcal{R}_{0 v}$ is the the number of mosquitoes that one human infects through the duration of the infectious period, which is also defined as

$$
\mathcal{R}_{0 v}=\sqrt{\frac{P \xi \epsilon \kappa \eta_{v}\left(\vartheta_{v}\left(1-\delta_{v}\right)-\left(\varrho+\mu_{v}\right)\right)}{\vartheta_{v}\left(1-\delta_{v}\right)\left(\varrho+\mu_{v}\right)\left(\eta_{v}+\varrho+\mu_{v}\right)}} .
$$

Furthermore, using Theorem 2 of [54], the following result is established.

2.2. Theorem. The disease free equilibrium, $\mathcal{E}_{0}$, of the model (2.1), is locally asymptotically stable $(L A S)$ if $\mathcal{R}_{0}<1$ and unstable otherwise. 
The basic reproduction number $\mathcal{R}_{0}$ measures the average number of new infections generated by a single infected individual in a completely susceptible population [5, 13, 54]. Thus, Theorem 2.2 implies that malaria can be eliminated from human population (when $\left.\mathcal{R}_{0}<1\right)$ if the initial sizes of the sub-populations are in the basin of attraction of the DFE.

2.2.2. Existence of endemic equilibria. In search of an endemic equilibrium point of model (2.1) (that is, equilibria where at least one of the infected components in model (2.1) is non-zero), we take the following steps.

Let $\mathcal{E}_{1}=\left(S_{h}^{* *}, E_{h}^{* *}, I_{h}^{* *}, R_{h}^{* *}, S_{v}^{* *}, E_{v}^{* *}, I_{v}^{* *}\right)$ represent any arbitrary endemic equilibrium of model (2.1). Also let

$$
\beta_{h}^{* *}=\frac{v \epsilon \kappa I_{v}^{* *}}{N_{h}^{* *}}, \quad \text { and } \quad \xi_{v}^{* *}=\frac{\xi \epsilon \kappa I_{h}^{* *}}{N_{h}^{* *}}
$$

be the forces of infection of human and vectors at steady state, respectively. Setting the right hand side of equation (2.1) to zero gives the following expressions.

$$
\begin{aligned}
& S_{h}^{* *}=\frac{\Phi_{h}+q R_{h}^{* *}}{\beta_{h}^{* *}+\mu_{h}}, \\
& E_{h}^{* *}=\frac{\beta_{h}^{* *} S_{h}^{* *}}{\eta_{h}+\mu_{h}}, \\
& I_{h}^{* *}=\frac{\eta_{h} E_{h}^{* *}}{\bar{\mu}}, \\
& R_{h}^{* *}=\frac{(\alpha+\varsigma) I_{h}^{* *}}{q+\mu_{h}}, \\
& S_{v}^{* *}=\frac{P \mu_{v}\left(\vartheta_{v}\left(1-\delta_{v}\right)+\left(\varrho+\mu_{v}\right)\right)}{\vartheta_{v}\left(1-\delta_{v}\right)\left(\left(\varrho+\mu_{v}\right)+\xi^{* *}\right)}, \\
& E_{v}^{* *}=\frac{\xi_{v}^{* *} S_{v}^{* *}}{\eta_{v}+\varrho+\mu_{v}}, \\
& I_{v}^{* *}=\frac{\eta_{v} E_{v}^{* *}}{\varrho+\mu_{v}} .
\end{aligned}
$$

Substituting (2.8) in (2.7), and we have,

$$
\xi_{v}^{* *}=\frac{\epsilon \kappa \xi \beta_{h}^{* *} \eta_{h} \mu_{h}\left(q+\mu_{h}\right) \Phi_{h}}{\left((\bar{\mu}) \mu_{h}\left(q+\mu_{h}\right)\left(\eta_{h}+\mu_{h}\right)+\beta_{h}^{* *}\left((\bar{\mu}) \mu_{h}\left(q+\mu_{h}\right)+\eta_{h}\left(q(\gamma+\mu)+(\bar{\mu}) \mu_{h}\right)\right)\right)-\left(\gamma \beta_{h}^{* *} \eta_{h}\left(q+\mu_{h}\right) \Phi_{h}\right) \Phi_{h}}
$$

It can be shown that the non-zero equilibria of the model satisfy the following polynomial (in terms of $\beta_{h}^{* *}$ )

$$
\left.P\left(\beta_{h}^{* *}\right)=A\left(\beta_{h}^{* *}\right)^{3}+B\left(\beta_{h}^{* *}\right)^{2}+C\left(\beta_{h}^{* *}\right)\right)
$$

$$
A\left(\beta_{h}^{* *}\right)^{2}+B\left(\beta_{h}^{* *}\right)+C=0,
$$


where,

$$
\begin{aligned}
A= & \vartheta_{v}\left(1-\delta_{v}\right)\left[(\bar{\mu}) \mu_{h}\left(q+\mu_{h}\right)+\eta_{h}\left(q \mu_{h}+\left(\alpha+\mu_{h}\right) \mu_{h}\right)\right]\left(\eta_{v}+\mu_{v}\right) \\
& {\left[(\bar{\mu}) \mu_{h}\left(q+\mu_{h}\right) \mu_{v}+\eta_{h}\left(\epsilon \kappa \xi \mu_{h}\left(q+\mu_{h}\right)+\left(q \mu_{h}+\left(\alpha+\mu_{h}\right) \mu_{h}\right)\left(\varrho+\mu_{v}\right)\right)\right] \Phi_{h} } \\
B= & \mu_{h}\left(q+\mu_{h}\right) \vartheta_{v}\left(1-\delta_{v}\right)(\bar{\mu})\left(\eta_{h}+\mu_{h}\right)\left(\eta_{v}+\varrho+\mu_{v}\right) \Phi_{h}\left[\mathcal{R}_{k}-\mathcal{R}_{0}^{2}\right] G_{1} \\
C= & (\bar{\mu})^{2} \mu_{h}^{2}\left(q+\mu_{h}\right)^{2}\left(\eta_{h}+\mu_{h}\right)^{2} \vartheta_{v}\left(1-\delta_{v}\right)\left(\eta_{v}+\varrho+\mu_{v}\right) \Phi_{h}\left[1-\mathcal{R}_{0}^{2}\right],
\end{aligned}
$$

where,

$$
\mathcal{R}_{k}=\frac{\epsilon \kappa \xi \eta_{h} \mu_{h}\left(q+\mu_{h}\right)+2\left[(\bar{\mu}) \mu_{h}\left(q+\mu_{h}\right)+\eta_{h}\left(q \mu_{h}+(\bar{\mu})\right]\left(\varrho+\mu_{v}\right)\right.}{\left(\varrho+\mu_{v}\right)\left[(\bar{\mu}) \mu_{h}\left(q+\mu_{h}\right) \eta_{h}\left(q\left(\gamma+\mu_{h}\right)+(\bar{\mu}) \mu_{h}\right)\right]}
$$

and

$$
G_{1}=(\bar{\mu}) \mu_{h}\left(q+\mu_{h}\right) \eta_{h}\left(q\left(\gamma+\mu_{h}\right)+(\bar{\mu}) \mu_{h}\right) .
$$

Note that the coefficient $A$ is always positive and $C$ (resp. $B$ ) is positive if $\mathcal{R}_{0}$ is less than 1 (resp $\left.\sqrt{\mathcal{R}_{k}}\right)$ respectively.

We have the following results:

\subsection{Corollary.}

(1) If $\mathcal{R}_{k} \geq 1$ then system (2.1) exhibits a forward bifurcation.

(2) If $\mathcal{R}_{k}<1$ then system (2.1) exhibits a backward bifurcation.

Proof.

(1) For $\mathcal{R}_{k} \geq 1$ we obtain when $\mathcal{R}_{0}>1$ that $C<0$. This implies that system (2.1) has a unique endemic steady state. If $\mathcal{R}_{0} \leq 1$, then $C \geq 0$ and $B \geq 0$. In this case system (2.1) has no endemic steady states.

(2) For $\mathcal{R}_{k}<1$ we discuss the following cases:

i. $\mathcal{R}_{0}>1$, in this case $C<0$ and system (2.1) has a unique endemic steady state.

ii. $\mathcal{R}_{0} \leq \sqrt{\mathcal{R}}_{k}$, in this case both $B$ and $C$ are positive implying that system (2.1) has no endemic steady states.

iii. $\sqrt{\mathcal{R}}_{k}<\mathcal{R}_{0}<1$, here $C>0$ and $B<0$ while the discriminant of (2.10), $\Delta\left(\mathcal{R}_{0}\right):=B^{2}-4 A C$, can be either positive or negative. We have $\Delta(1)=$ $B^{2}>0$ and $\Delta\left(\sqrt{\mathcal{R}}_{k}\right)=-4 A C<0$, then there exists $R_{0 c}$ such that $\Delta\left(R_{0 c}\right)=0$, $\Delta\left(\mathcal{R}_{0}\right)<0$ for ${\sqrt{\mathcal{R}_{k}}}<\mathcal{R}_{0}<R_{0 c}$ and $\Delta\left(\mathcal{R}_{0}\right)>0$ for $R_{0 c}<\mathcal{R}_{0}$. This together with the signs of $B$ and $C$ imply that system (2.1) has no endemic steady states when $\sqrt{\mathcal{R}}_{k}<\mathcal{R}_{0}<R_{0 c}$, one endemic steady state when $\mathcal{R}_{0}=R_{0 c}$ and two endemic steady states when $R_{0 c}<\mathcal{R}_{0}<1$.

\subsection{Model analysis with climate-dependent parameters.}

2.3.1. Study case and data. Over the years, Plasmodium falciparum has been identified as the main causes of malaria in three northeastern provinces of South Africa namely; Mpumalanga, KwaZulu-Natal and Limpopo [10,59]. In this study, we validate our model against the malaria transmission case in Limpopo Province. The province is situated in the northernmost corner of South Africa (as in Fig. 2). It experiences long sunny days and dry weather on most days with high rainfall between October - March every year [28] as shown in Fig. 3. In Fig. 3a, four peaks with three nadirs are observed for temperature between January 2002 - December 2004. The peaks occur between December-February while the nadirs falls within June - August each year. Similar patterns are observed for rainfall in Fig. 3b. This implies that the province simultaneously experiences high 


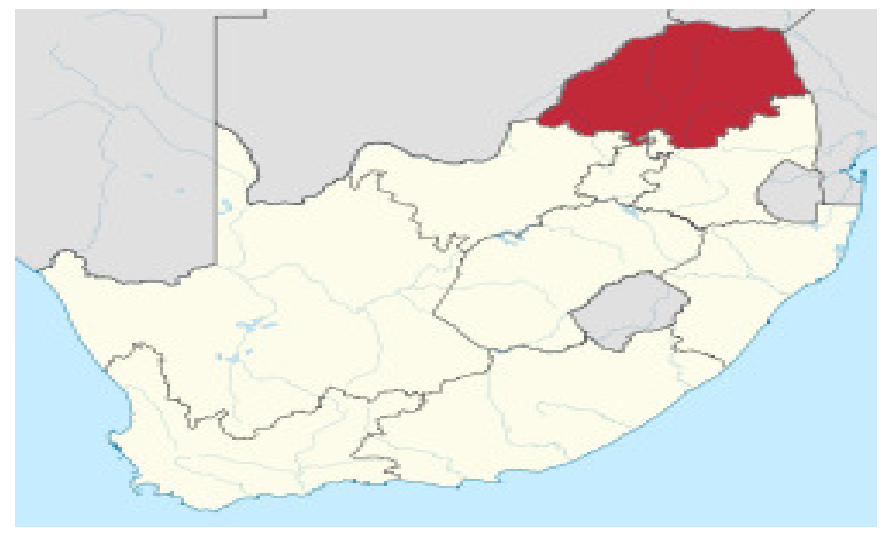

Figure 2. Map of South Africa showing Limpopo province (in red). Source: http://en.wikip-edia.org/wiki/Limpopo

temperature and rainfall between December - February with low temperature and rainfall between June-August every year. Also, malaria transmission in this province is seasonal with climatic conditions affecting the development of mosquitoes and malaria parasites $[6,47]$. Hence, we limit our study on the effects of temperature and rainfall on the transmission of malaria in Limpopo province. The input climate data used for this study is obtained from the South African Weather Service (SAWS). The data consists of daily rainfall, minimum and maximum temperature that spans the period of 2002 to 2004, while the monthly malaria cases for the same period were obtained from the South African Department of Health.

2.3.2. Parameter estimates. Some of the parameters relevant to our study has also featured in previous studies of malaria transmission modeling such as in $[8,32,39,59]$. In these papers the authors have obtained fairly good numerical values for these parameters and we reference such parameter values in Table 1. Following the approach of [59], we estimate below the death and birth rate of humans in Limpopo Province.

Human death rate $\mu_{h}$. According to the World Health Organization (WHO) report, the life expectancy for South Africa in 2002 was 49.1 years. Hence, the human death rate is calculated as

$$
\mu_{h}=\frac{1}{49.1 \times 365}=0.000056 \mathrm{Day}^{-1}
$$

Human birth rate $\Phi_{h}$. The South Africa Census for 2001 stated that the total human population for Limpopo province was 5,273,642 [46]. Therefore, the human birth rate for Limpopo province is estimated as

$$
\Phi_{h}=\mu_{h} \times 5,273,642=295 \text { human } / \text { day }
$$

The climate-dependent parameters affect the processes occurring during either the larva or adult stage. For instance, adequate rainfall is essential for survival of eggs, larva and pupae survival, while temperature is influential in the gonotropic cycle, a period between blood meal and oviposition [4]. 
(a)

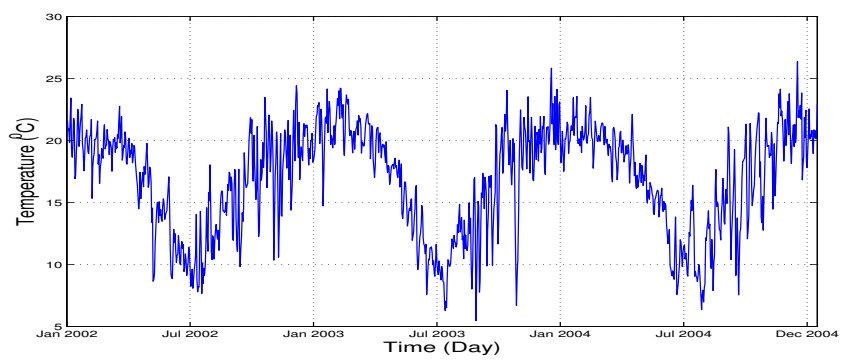

(b)

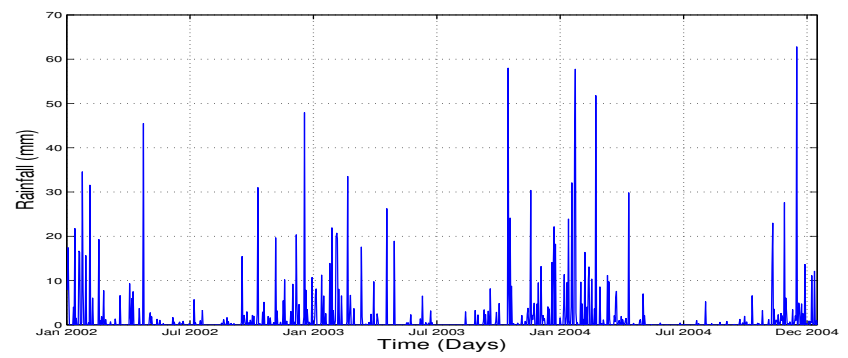

Figure 3. (a) Daily mean temperature and (b) rainfall of Limpopo province between January 2002 - December 2004.

Mosquito birth rate. Aside climatic factors, several studies (e.g. [2, 45, 53]) have explained that the availability and conditions of mosquito breeding sites (such as puddles, ponds, containers) play a crucial role in determining the oviposition rate. For instance, it has been established that some mosquitoes will not lay egg if there is no available breeding site $[29,50]$. It is also possible for female mosquitoes to distribute their eggs among suitable and unoccupied container [55]. Based on these facts, we adopt adopt the mosquito birth rate of [43], as

$$
\vartheta_{v}(T, R)=\frac{n_{e} p_{e}(R) p_{l}(R, T) p_{p}(R)}{t_{e}+t_{l}(T)+t_{p}}
$$

where $n_{e}$ is the number of eggs laid per adult mosquito per oviposition, $p_{e}(R)$, $p_{l}(R, T)$ and $p_{p}(R)$ are the survival probabilities of eggs, larvae and pupae respectively. The duration of each development stage is given as $t_{e}, t_{l}(T)$ and $t_{p}$ and the average larval duration $t_{l}(T)=1 /(0.0554 T-0.06737)$. We assume that temperature and rainfall act independently on the survival probability $p_{l}$ of larvae (cf. [43]) such that $p_{l}(R, T)=$ $\pi_{1}(T) \pi_{2}(R)$. In this product the first factor is taken as

$$
\pi_{1}(T)=e^{-(0.0554 T-0.06737)} \text {. }
$$

Rainfall has been shown to positively correlate with malaria incidence [42], although excessive rainfall may flush out larvae and breeding sites [12, 53]. Hence we assume a quadratic relationship between the survival probabilities of eggs, larvae and pupae and rainfall. For larvae, we assume

$$
\pi_{2}(R)=\left[\frac{4 p_{l}^{*}}{R_{l}^{2}}\right] R\left(R_{l}-R\right),
$$

where $R_{l}$ is the rainfall limit beyond which breeding site get flushed out and no immature stages survive [43]. The constant $p_{l}^{*}$ is the maximum survival probability. 
Mosquito mortality rate. To estimate the temperature-dependent mortality rate of adult mosquito, we adopt the expression of $[4,33,38]$ that;

$$
\mu_{v}(T)=\frac{1}{\left(-4.4+1.31 T-0.03 T^{2}\right)} \text { Day }^{-1}
$$

Larval carrying capacity. We assume that rainfall generates available water for larval development, and that the carrying capacity $P$ evolves according to a dynamical equation that takes into account habitable per unit area and unit time [4]. It is measured in terms of an effective maximum number of larvae available in a given region and we say

$$
P=\frac{P_{A}}{P_{E}} R
$$

where $P_{E}=10^{-3}<P_{E}<10^{0}$ is the carrying capacity decaying rate, and the conversion factor $P_{A}$ varies between 0 and $10^{4}$. Other parameters are shown in Table 1 with references.

\section{Results and discussion}

3.1. Effects of temperature-dependent parameters. In this section, we illustrate the relationship between temperature and mosquito death rate, biting rate, progression rate and reproduction number in Fig. 4. The U-curve in Fig. 4a explains in line with $[17,38,44]$, that the mosquito death rate is high at low temperature (below $18^{\circ} \mathrm{C}$ ), low between $18-25^{\circ} \mathrm{C}$ and increases at temperature beyond $25^{\circ} \mathrm{C}$. Fig. $4 \mathrm{~b}$ and Fig. $4 \mathrm{~d}$ show unimodal curves (as also appeared in $[36,38]$ ) which are thermally constrained at both low and high temperature. Fig. 4b demonstrates low mosquito biting rate at temperatures below $12^{\circ} \mathrm{C}$, gradually increasing with a slight declination at upper thermal limit of $35^{\circ} \mathrm{C}$. Although the limit is considerably different from that obtained in the study [7] which is $30^{\circ} \mathrm{C}$, it matches the findings of $[36,38]$ which is $35^{\circ} \mathrm{C}$. However, the reproduction number $\mathcal{R}_{0}$ in Fig. 4 d indicates that the optimal temperature for malaria transmission is $30^{\circ} \mathrm{C}$ with transmission occurring between $13^{\circ} \mathrm{C}-40^{\circ} \mathrm{C}$, and with threshold where $\mathcal{R}_{0}>1$ between $18^{\circ} \mathrm{C}-38^{\circ} \mathrm{C}$. The transmission range is closer to the new estimate of [36] which is $15^{\circ} \mathrm{C}-35^{\circ} \mathrm{C}$, while the optimal temperature and the threshold is similar to the findings of [38] which are $31.5^{\circ} \mathrm{C}$ and $22.34^{\circ} \mathrm{C}-38.6^{\circ} \mathrm{C}$ respectively. Over a certain temperature interval, the parasite development rate in Fig. 4c increases with temperature.

In Fig. 5, the incidence cases of malaria infection is simulated by our model and compared with malaria monthly cases for Limpopo province as reported by South African Department of Health. The figure shows a good fit between the observed and predicted incidence over wide range of time. It can be seen in both results that malaria is climatedriven with epidemic peaks between December-February when temperature and rainfall is high in the province (as shown in Fig. 3). The results indicate a seasonal pattern as both curves decrease progressively from February through August and then gradually increase from September through January. However, we observe high temperature and rainfall in December 2002 resulting in an epidemic peak (in Fig. 5) in January 2003. With ideal temperature $\left(28^{\circ} \mathrm{C}\right)$ and conditions, the development of Anopheles from juvenile to adult stage takes about 14 days $[12,21,53]$, while symptoms of falciparum malaria arise between 7-15 days depending on immunity of the host [15].

3.2. Spatial distribution of malaria reproduction number over Limpopo. In rainy summer, we assume several water pools serving as mosquito breeding sites are generated by rainwater. This allows mosquitoes to lay their eggs, which later develop to adult mosquitoes if the pools are sustained for at least 14 days. We also assumed that additional pools are intentionally created for the purpose of cattle watering, irrigation 
(a)
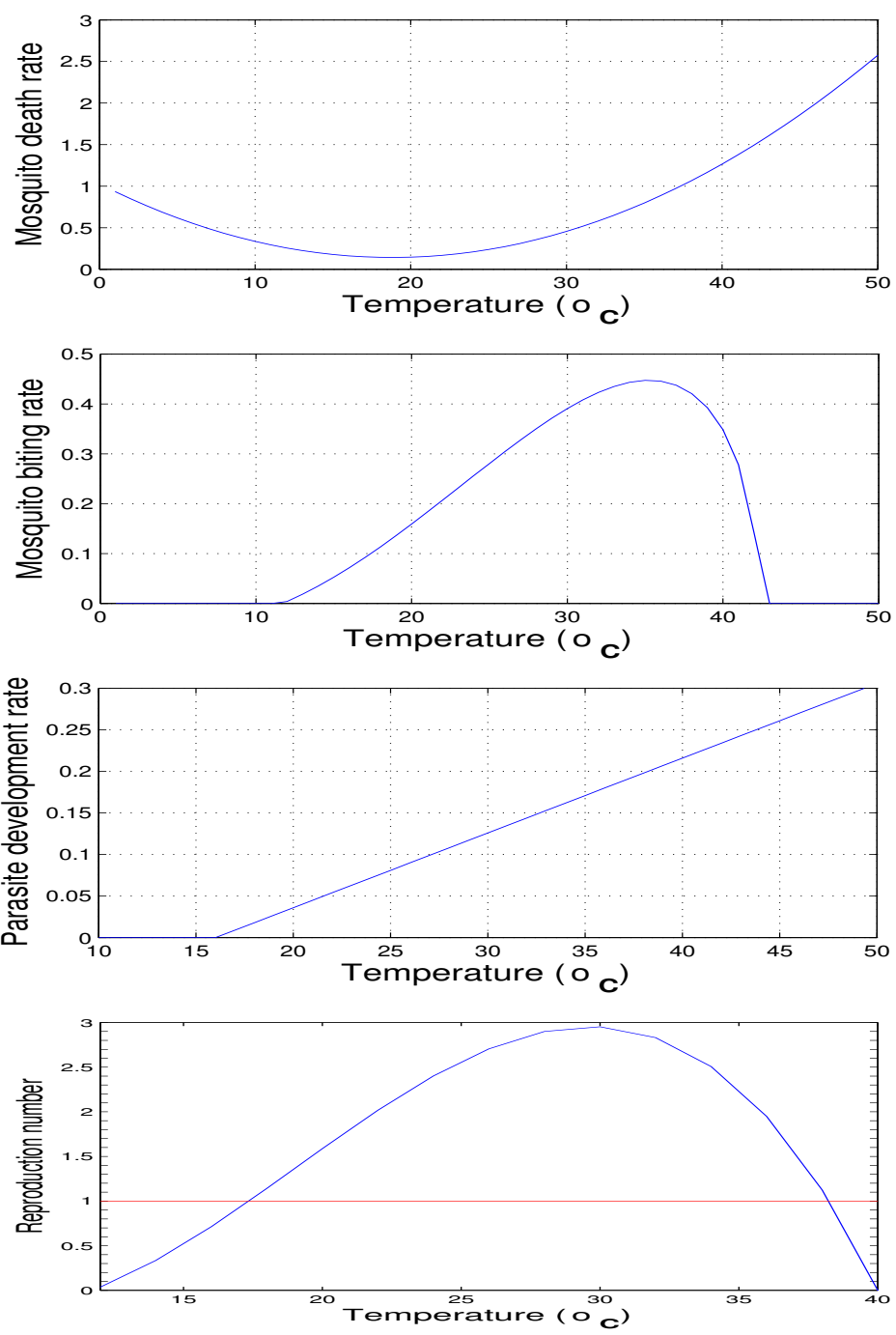

Figure 4. Simulation of (a) mosquito death rate, (b) mosquito biting rate, (c) progression rate of mosquitoes, and (d) $\mathcal{R}_{0}$ versus temperature.

and so on [58]. Using our model with Observational-Reanalysis hybrid datasets for daily temperature, we numerically calculate a time-varying approximation to the basic reproduction number $\left(\hat{\mathcal{R}}_{0}\right)$ over Limpopo between December 2002 and December 2003 with the assumption that $\mathcal{R}_{0}$ varies with time. To achieve this, we incorporate the climate-dependent parameters to expression (2.4). The 1.0-degree spatial resolution and Global Meteorological Forcing Dataset for land surface modelling, are produced by the Terrestrial Hydrology Research Group at Princeton University (hereafter, [48]). The results in Fig. 6 and Fig. 7 show that malaria transmission in South Africa is distinctly seasonal, with transmission limited to the warm and rainy summer months (September to May) with very low cases in June, July and August [6]. We further investigate the 


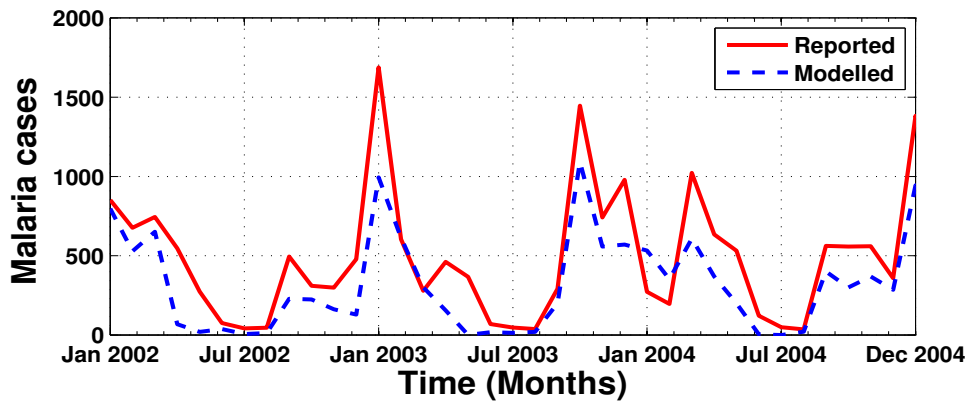

Figure 5. The reported cases and modeled cases for Limpopo province, South Africa 2002 - 2004.

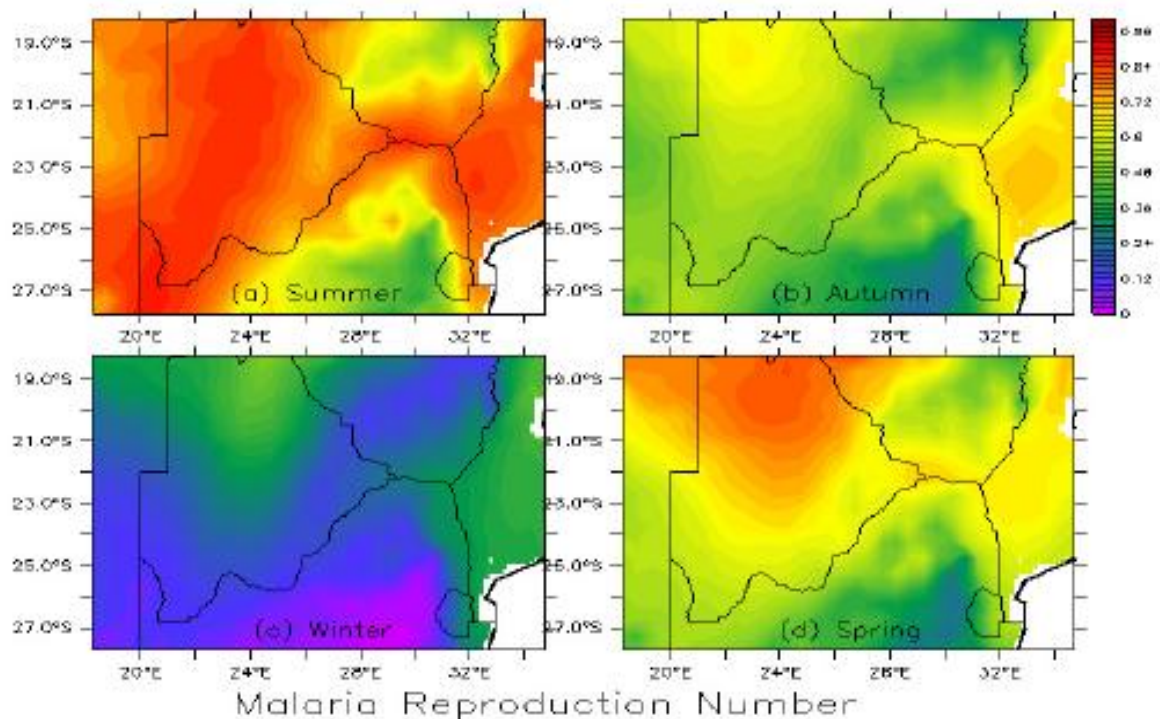

Figure 6. Simulation of basic reproduction number when $\delta_{v}$ is low $\left(\delta_{v}=0.2\right)$.

impact of larval population abundance on reproduction number by considering a low and high proportion of larva that died in the process of becoming adult. It is observed in Fig. 6 that when the proportion $\delta_{v}=0.2$, the maximum value of $\hat{\mathcal{R}}_{0}$ over Limpopo, which is found in summer is 0.84 . The value reduces to 0.48 when $\delta_{v}=0.9$. This implies that when $\delta_{v}$ is low, it increases the proportion of larvae $\left(1-\delta_{v}\right)$ that made it to the adult stage, thus increases the number of mosquitoes available for transmission. On the other hand, high $\delta_{v}$ reduces the proportion of adult mosquitoes available for transmission, thus reduces the reproduction number. 


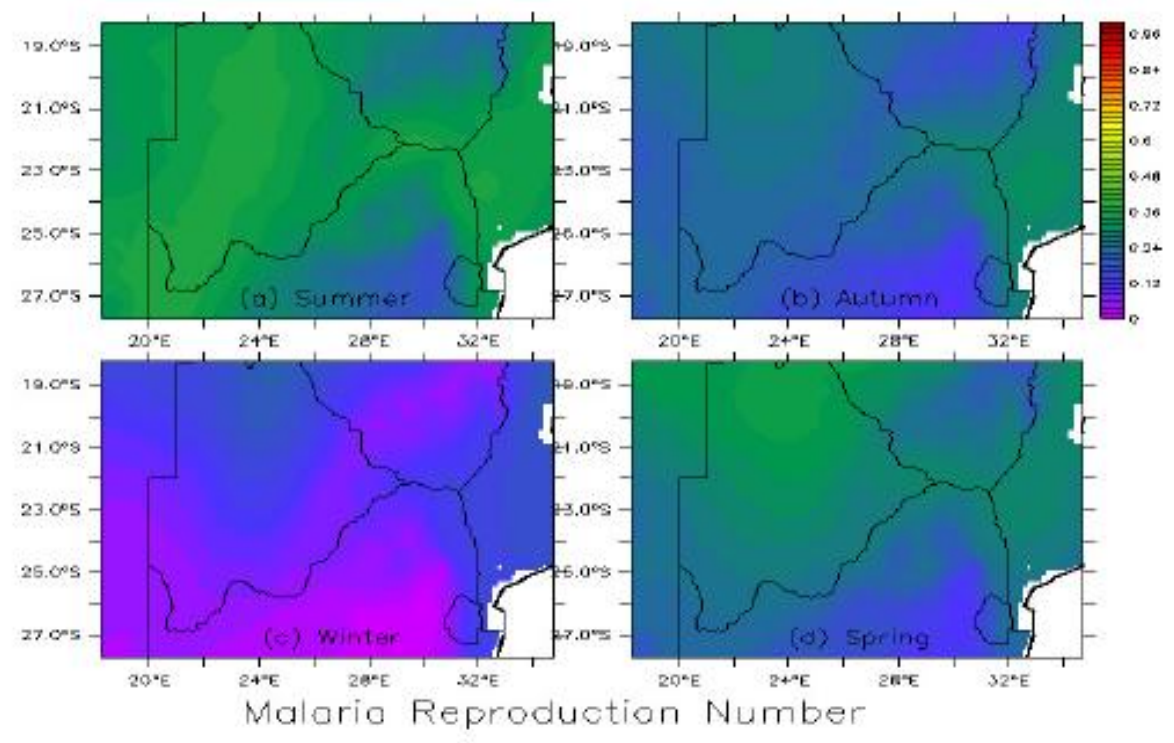

Figure 7. Simulation of basic reproduction number when $\delta_{v}$ is high $\left(\delta_{v}=0.9\right)$.

\section{Conclusion}

In this study, a deterministic malaria model to explore the impact of temperature and rainfall on malaria transmission is presented and analysed. We derived the basic reproduction number and examined the model for the existence of disease-free and endemic equilibrium points. The system has an equilibrium point in which the disease persist in the population.

In Section 4, we examined the effect of temperature on mosquito death, biting rate, parasite development rate and reproduction number. It is verified that parasite development rate increases along with temperature, while mosquito biting rate and reproduction number are thermally constrained at both low and high temperature.

The model is validated against malaria transmission in Limpopo Province, South Africa for 2002 - 2004 in Section 5. The results indicate that malaria transmission in the province is seasonal with epidemic peak between December-February when temperature and rainfall are relatively high. We further investigate the effect of larva death rate on the reproduction number over entire South Africa for 2003. Our findings show that a high rate of larva mortality reduces the reproduction number, and increases it when low. This suggests that destruction of mosquitoes breeding sites and regular use of larvicides have high potential to reduce malaria transmission. Further activities like spraying, use of treated bed nets that contribute to mosquitoes death should be encouraged mostly between September and May when the climatic conditions are favourable for mosquitoes' development in South Africa.

However, there are other factors aside climatic reasons which need to be considered in studying malaria transmission. Some of these factors include; migration of infected human, economic development and so on. We leave these aspects for future studies. 
Table 1. Parameters of the malaria model 2.1

\begin{tabular}{|c|c|c|c|}
\hline Description & Symbol & Value & Ref \\
\hline Mosquito biting rate & $\epsilon(T)$ & $\begin{array}{l}0.000203 D_{1} D_{2} \\
D_{1}=T^{2}-11.7 T \\
D_{2}=\sqrt{42.3-T}\end{array}$ & {$[38,41]$} \\
\hline Adult mosquito death rate & $\mu_{v}(T)$ & $\frac{1}{\left(-4.4+1.31 T-0.03 T^{2}\right)}$ & {$[4,38,33]$} \\
\hline Progression rate of mosquitoes & $\eta_{v}(T)$ & $\frac{\left(T-T_{\min }\right)}{111}$ & {$[30,38,43]$} \\
\hline Duration of egg development & $t_{e}$ & 1 (days) & {$[43]$} \\
\hline Duration of pupa development & $t_{p}$ & 1 (days) & {$[43]$} \\
\hline Max. survival probability of egg & $p_{e}^{*}$ & 0.9 & {$[43]$} \\
\hline Max. survival probability of pupa & $p_{p}^{*}$ & 0.25 & {$[43]$} \\
\hline Max. survival probability of larva & $p_{l}^{*}$ & 0.75 & {$[43]$} \\
\hline Rainfall limit to flushing & $R_{l}$ & $50(\mathrm{~mm})$ & {$[43]$} \\
\hline $\begin{array}{l}\text { Num. of eggs laid per adult per ovip. } \\
\text { Min. temperature for } P \text {. falciparum }\end{array}$ & $n_{e}$ & 200 & {$[38,43]$} \\
\hline survival & $T_{\min }$ & $16\left({ }^{\circ} C\right)$ & {$[43]$} \\
\hline Carrying capacity of larva & $\mathrm{P}$ & 1000000 & Est. \\
\hline Induced death rate & $\gamma$ & 0.0004 & {$[9,38]$} \\
\hline Loss of immunity & $\alpha$ & 0.00014 & {$[38]$} \\
\hline Recovery rate of humans & $q$ & 0.005 & {$[9,38]$} \\
\hline $\begin{array}{l}\text { Progression rate of humans from the } \\
\text { exposed class to infectious class }\end{array}$ & $\eta_{h}$ & $1 / 14$ & {$[9,38]$} \\
\hline Natural death rate of humans & $\mu_{h}$ & 0.000056 & Est. \\
\hline Rate of treatment & $\varrho$ & $0.01-0.7$ & Est. \\
\hline $\begin{array}{l}\text { Probability of transmission of } \\
\text { infection from an infectious human }\end{array}$ & & & \\
\hline to a susceptible mosquito & $v$ & 0.09 & {$[38,43]$} \\
\hline $\begin{array}{l}\text { Probability of transmission of infection } \\
\text { from an infectious mosquito to a } \\
\text { susceptible human }\end{array}$ & $\xi$ & 0.04 & {$[38,43]$} \\
\hline Birth rate of humans & $\Phi_{h}$ & 295 human/day & Est. \\
\hline Contact rate & $\kappa$ & 0.6 & {$[32]$} \\
\hline Carying capacity decaying rate & $P_{E}$ & 0.01 & {$[43]$} \\
\hline Conversion factor & $P_{A}$ & 1000 & {$[43]$} \\
\hline
\end{tabular}

Acknowledgements. This research was carried out for the iDEWS (infectious Diseases Early-Warning System) project supported by SATREPS (Science and Technology Research Partnership for Sustainable Development) Program of JICA (JAPAN International Cooperation Agency)/AMED (Japan Agency for Medical Research and Development) in Japan and the ACCESS (Applied Centre for Climate and Earth Systems Science) program of NRF (National Research Foundation) and DST(Department of Science and Technology in South Africa). 
The author (OKO) acknowledges, with thanks, the financial support of NRF, South Africa through research grant: 74816 .

\section{References}

[1] Abiodun, G.J., Marcus, N., Okosun, K.O. and Witbooi, P.J. A model for control of $H I V / A I D S$ with parental care, International Journal of Biomathematics 6 (02), 1350006, 2013.

[2] Abiodun, G.J., Maharaj, R., Witbooi, P. and Okosun, K.O. Modelling the influence of temperature and rainfall on the population dynamics of Anopheles arabiensis, Malaria Journal 15 (1), 364, 2016.

[3] Abiodun, G.J., Witbooi, P. and Okosun, K.O. Modeling and analyzing the impact of temperature and rainfall on mosquito population dynamics over KwazuluNatal province, South Africa, International Journal of Biomathematics 2016. DOI: http://dx.doi.org/10.1142/S1793524517500553.

[4] Alonso, D., Bouma, M.J. and Pascual, M. Epidemic malaria and warmer temperatures in recent decades in an East African highland, Proceedings of the Royal Society of London B: Biological Sciences 278 (1712), 1661-1669, 2011.

[5] Anderson, R.M., May, R.M. and Anderson, B. Infectious diseases of humans: dynamics and control (Vol. 28). Oxford: Oxford university press, 1992.

[6] Gerritsen, A.A., Kruger, P., van der Loeff, M.F.S. and Grobusch, M.P. Malaria incidence in Limpopo Province, South Africa, 1998-2007, Malaria journal 7 (1), 162, 2008.

[7] Briere, J.F., Pracros, P., Le Roux, A.Y. and Pierre, J.S. A novel rate model of temperaturedependent development for arthropods, Environmental Entomology 28 (1), 22-29, 1999.

[8] Chitnis, N., Hyman, J.M. and Cushing, J.M. Determining important parameters in the spread of malaria through the sensitivity analysis of a mathematical model, Bulletin of mathematical biology $\mathbf{7 0}$ (5), 1272, 2008.

[9] Chiyaka, C., Tchuenche, J.M., Garira, W. and Dube, S. A mathematical analysis of the effects of control strategies on the transmission dynamics of malaria, Applied Mathematics and Computation 195 (2), 641-662, 2008.

[10] Craig, M.H., Kleinschmidt, I., Nawn, J.B., Le Sueur, D. and Sharp, B.L. Exploring 30 years of malaria case data in KwaZulu-Natal, South Africa: part I. The impact of climatic factors, Tropical Medicine \& International Health 9 (12), 1247-1257, 2004.

[11] Craig, M.H., Snow, R.W. and Le Sueur, D. A climate-based distribution model of malaria transmission in sub-Saharan Africa, Parasitology today 15 (3), 105-111, 1999.

[12] Depinay, J.M.O., Mbogo, C.M., Killeen, G., Knols, B., Beier, J., Carlson, J., Dushoff, J., Billingsley, P., Mwambi, H., Githure, J. and Toure, A.M. A simulation model of African Anopheles ecology and population dynamics for the analysis of malaria transmission, Malaria journal 3 (1), 29, 2004.

[13] Diekmann, O., Heesterbeek, J.A.P. and Roberts, M.G. The construction of next-generation matrices for compartmental epidemic models, Journal of the Royal Society Interface, p.rsif20090386, 2009.

[14] Eckhoff, P.A. A malaria transmission-directed model of mosquito life cycle and ecology, Malaria journal, 10 (1), 303, 2011.

[15] Ermert, V., Fink, A.H., Jones, A.E. and Morse, A.P. Development of a new version of the Liverpool Malaria Model. I. Refining the parameter settings and mathematical formulation of basic processes based on a literature review, Malaria journal 10 (1), 35, 2011.

[16] Ermert, V., Fink, A.H., Jones, A.E. and Morse, A.P. Development of a new version of the Liverpool Malaria Model. II. Calibration and validation for West Africa, Malaria journal 10 (1), 62, 2011.

[17] Rubel, F. and Brugger, K. Dynamics of infectious diseases according to climate change: the Usutu virus epidemics in Vienna, In Game meat hygiene in focus 173-198, 2011.

[18] Hethcote, H.W. The mathematics of infectious diseases, SIAM review 42 (4), 599-653, 2000.

[19] Hoshen, M.B. and Morse, A.P. A weather-driven model of malaria transmission, Malaria Journal 3 (1), 32, 2004. 
[20] Yang, H.M. Malaria transmission model for different levels of acquired immunity and temperature-dependent parameters (vector), Revista de saude publica 34 (3), 223-231, 2000.

[21] Jepson, W.F., Moutia, A. and Courtois, C. The malaria problem in Mauritius: the bionomics of Mauritian anophelines, Bulletin of entomological research 38 (01), 177-208, 1947.

[22] Li, J., A malaria model with partial immunity in humans, Mathematical biosciences and engineering 5 (4), 789-801, 2008.

[23] Joshi, H.R. Optimal control of an HIV immunology model, Optimal control applications and methods 23 (4), 199-213, 2002.

[24] Jones, A.E. and Morse, A.P. Application and validation of a seasonal ensemble prediction system using a dynamic malaria model, Journal of Climate 23 (15), 4202-4215, 2010.

[25] Jones, A.E. and Morse, A.P. Skill of ENSEMBLES seasonal re-forecasts for malaria prediction in West Africa, Geophysical Research Letters 39 (23), 2012.

[26] Koella, J.C. On the use of mathematical models of malaria transmission, Acta tropica 49 (1), 1-25, 1991.

[27] Lafferty, K.D. The ecology of climate change and infectious diseases, Ecology 90 (4), 888900, 2009.

[28] Limpopo Province, South Africa. SouthAfrica.info. http://www.southafrica.info/about/geography/limpopo.htm.UxHXN85j-18 (Feb 2014).

[29] Maharaj, R. Life table characteristics of Anopheles arabiensis (Diptera: Culicidae) under simulated seasonal conditions, Journal of medical entomology 40 (6), 737-742, 2003.

[30] Macdonald, G. The epidemiology and control of malaria, 1957, London, New York, and Toronto: Oxford University Press Google Scholar.

[31] MacDonald, G., Cuellar, C.B. and Foll, C.V. The dynamics of malaria, Bulletin of the World Health Organization 38 (5), 743, 1968.

[32] Makinde, O.D. and Okosun, K.O. Impact of chemo-therapy on optimal control of malaria disease with infected immigrants, BioSystems 104 (1), 32-41, 2011.

[33] Martens, W.J., Niessen, L.W., Rotmans, J., Jetten, T.H. and McMichael, A.J. Potential impact of global climate change on malaria risk Environmental health perspectives 103 (5), 458, 1995.

[34] McKenzie, F.E. Why model malaria?, Parasitology Today 16 (12), 511-516, 2000.

[35] Moghadas, S.M. and Gumel, A.B. Global stability of a two-stage epidemic model with generalized non-linear incidence, Mathematics and computers in simulation 60 (1), 107-118, 2002.

[36] Mordecai, E.A., Paaijmans, K.P., Johnson, L.R., Balzer, C., Ben-Horin, T., Moor, E., McNally, A., Pawar, S., Ryan, S.J., Smith, T.C. and Lafferty, K.D. Optimal temperature for malaria transmission is dramatically lower than previously predicted, Ecology letters $\mathbf{1 6}$ (1), 22-30, 2013.

[37] Nakazawa, M., Ohmae, H., Ishii, A. and Leafasia, J. Malaria infection and human behavioral factors: A stochastic model analysis for direct observation data in the Solomon Islands, American journal of human biology, 10 (6), 781-789, 1998.

[38] Ngarakana-Gwasira, E.T., Bhunu, C.P. and Mashonjowa, E. Assessing the impact of temperature on malaria transmission dynamics, Afrika Matematika 25 (4), 1095-1112, 2014.

[39] Okosun, K.O. and Makinde, O.D. Modelling the impact of drug resistance in malaria transmission and its optimal control analysis, International Journal of Physical Sciences 6 (28), 6479-6487, 2011.

[40] Ozair, M., Lashari, A.A., Jung, I.H. and Okosun, K.O. Stability analysis and optimal control of a vector-borne disease with nonlinear incidence, Discrete Dynamics in Nature and Society, 2012.

[41] Paaijmans, K.P., Cator, L.J. and Thomas, M.B. Temperature-dependent pre-bloodmeal period and temperature-driven asynchrony between parasite development and mosquito biting rate reduce malaria transmission intensity PLOS one 8 (1), e55777, 2013.

[42] Paaijmans, K.P., Wandago, M.O., Githeko, A.K. and Takken, W. Unexpected high losses of Anopheles gambiae larvae due to rainfall, PLoS One 2 (11), e1146, 2007.

[43] Parham, P.E. and Michael, E. Modelling climate change and malaria transmission, Modelling Parasite Transmission and Control 184-199, 2010. 
[44] Reisen, W.K. Effect of temperature on Culex tarsalis (Diptera: Culicidae) from the Coachella and San Joaquin valleys of California, Journal of medical entomology 32 (5), 636-645, 1995.

[45] Rausher, M.D. Larval habitat suitability and oviposition preference in three related butterflies, Ecology 60 (3), 503-511, 1979.

[46] South African National Census of 2001. http://www.statssa.gov.za/census01/html/.

[47] Silal, S.P., Barnes, K.I., Kok, G., Mabuza, A. and Little, F. Exploring the seasonality of reported treated malaria cases in Mpumalanga, South Africa, PloS one 8 (10), e76640, 2013.

[48] Sheffield, J., Goteti, G. and Wood, E.F. Development of a 50-year high-resolution global dataset of meteorological forcings for land surface modeling, Journal of Climate 19 (13), 3088-3111, 2006.

[49] Ruan, S., Xiao, D. and Beier, J.C. On the delayed Ross-Macdonald model for malaria transmission, Bulletin of mathematical biology 70 (4), 1098-1114, 2008.

[50] le Sueur, D. and Sharp, B.L. The breeding requirements of three members of the Anopheles gambiae Giles complex (Diptera: Culicidae) in the endemic malaria area of Natal, South Africa, Bulletin of entomological research 78 (04), 549-560, 1988.

[51] Smith, T.A. Estimation of heterogeneity in malaria transmission by stochastic modelling of apparent deviations from mass action kinetics, Malaria journal 7 (1), 12, 2008.

[52] Thomson, M.C., Doblas-Reyes, F.J., Mason, S.J., Hagedorn, R., Connor, S.J., Phindela, T., Morse, A.P. and Palmer, T.N. Malaria early warnings based on seasonal climate forecasts from multi-model ensembles, Nature 439 (7076), 576-579, 2006.

[53] Tompkins, A.M. and Ermert, V. A regional-scale, high resolution dynamical malaria model that accounts for population density, climate and surface hydrology, Malaria journal 12 (1), 65, 2013.

[54] Van den Driessche, P. and Watmough, J. Reproduction numbers and sub-threshold endemic equilibria for compartmental models of disease transmission, Mathematical biosciences 180 (1), 29-48, 2002.

[55] Wang, Y., Gilbreath III, T.M., Kukutla, P., Yan, G. and Xu, J. Dynamic gut microbiome across life history of the malaria mosquito Anopheles gambiae in Kenya, PloS one 6 (9), e24767, 2011.

[56] World Health Organization. World Malaria Report 2008. http://www.who.int/malaria/publications/world-malaria-report-2008/report/en/

[57] World Health Organization. World Malaria Report 2015. http://www.who.int/malaria/publications/world-malaria-report-2015/report/en/

[58] Yazoume, Y., Hoshen, M., Kyobutungi, C., Louis, V.R. and Sauerborn, R. Local scale prediction of Plasmodium falciparum malaria transmission in an endemic region using temperature and rainfall, Global Health Action 2, 2009.

[59] Lou, Y. and Zhao, X.Q. A climate-based malaria transmission model with structured vector population, SIAM Journal on Applied Mathematics 70 (6), 2023-2044, 2010. 
